\title{
Desarrollo de competencias emprendedoras como estrategias para el crecimiento personal en estudiantes de bachillerato del Distrito 09D08 de Guayaquil, 2021
}

\section{Development of entrepreneurial competencies as strategies for personal growth in high school students of District 09D08 of Guayaquil, 2021}

DOI: $10.46932 / \mathrm{sfjdv} 3 \mathrm{n} 1-029$

Received in: Dec 30st, 2021

Accepted in: Jan 1th, 2022

\author{
Aurora Victoria Gómez Quintana \\ Universidad César Vallejo \\ Maestra en Administración de la Educación \\ Docente de emprendimiento y gestion \\ La 22 entre el Oro y Vacas Galindo -Guayaquil-Ecuador \\ E-mail: vitocky1@hotmail.com \\ George Artemio Galarza Baque \\ Universidad César Vallejo \\ Maestro en Administración de la Educación \\ Docente de matemática y física \\ Mapasingue oeste Mz 1380 s. 14 -Guayaquil-Ecuador \\ E-mail: george_agb@hotmail.com \\ Álvaro Alberto Berruz Guerrero \\ Universidad César Vallejo \\ Maestro en Administración de la Educación \\ Docente de informática y telecomunicaciones \\ Florida norte Cooperativa unidos somos más mz. 384 v 4 -Guayaquil-Ecuador \\ E-mail: alvberruz4000@gmail.com

\section{Cecilia Gisela Soledispa Rodríguez} \\ Universidad César Vallejo \\ Maestra en Administración de la Educación \\ Docente de emprendimiento y gestion \\ Mapasingue oeste coop.9 de enero mz. E22 SOL.39 -Guayaquil-Ecuador \\ E-mail: cecilia_s19976@ hotmail.com \\ Jenny Patricia Barre Bustamante \\ Universidad César Vallejo \\ Maestra en Administración de la Educación \\ Docente de emprendimiento y gestion \\ Cdla. Guayacanes mz. 3 v 16-Guayaquil-Ecuador \\ E-mail: pattybarre@ hotmail.com
}

\section{RESUMEN}

La presente investigación: Desarrollo de competencias emprendedoras como estrategias para el crecimiento personal en estudiantes de bachillerato del Distrito 09D08 de Guayaquil, 2021 y su objetivo 
general Proponer un programa con el desarrollo de competencias emprendedoras que permitirán contribuir en el crecimiento personal de los estudiantes de bachillerato del distrito 09D08 de Guayaquil. El estudio se realizó con una población de 35 estudiantes y una muestra No probabilística, el tipo de investigación propositivo-descriptivo, enfoque cualitativo y diseño no experimental, se aplicó la técnica de la encuesta, y como instrumento un cuestionario constituido por veinte ítems, aplicando en cada una de ella cinco alternativas con la escala de valoración de Lickert. Los datos recolectados fueron procesados en el software SPSS, para la validación del cuestionario se realizó una matriz del contenido, fue validada mediante el juicio de 5 expertos, la confiabilidad fue de ,928, se utilizó el coeficiente de Alfa de Cronbach. Los resultados de la investigación mostraron que en general, el crecimiento personal de los estudiantes de bachillerato del Distrito 09D08 de una Institución Educativa de Guayaquil, es valorado como deficiente. Esto se evidencio a través de las dimensiones de la variable evaluada (Tabla 12), del 100\% de los estudiantes encuestados, $68.6 \%$ muestran dificultad para resolver conflictos, 84,30\% no son empáticos, $68.6 \%$ tienen una baja autoestima, $74,3 \%$ no tienen capacidad de influir en las personas, $68.5 \%$ muestran dificultad para la toma de decisiones, $68.60 \%$ muestran dificultad en el desarrollo de habilidades y competencias, $80.0 \%$ carece de autoconfianza, aun $74.20 \%$ no tienen deseos de superación. El programa propuesto de crecimiento personal a través del desarrollo de competencias emprendedoras como una alternativa para mejorar y superar las limitaciones de manera considerable y mejorar su bienestar

Palabras claves: relaciones interpersonales, empatía, toma de decisiones, autorrealización, liderazgo.

\begin{abstract}
This research: Development of entrepreneurial competencies as strategies for personal growth in high school students of District 09D08 of Guayaquil, 2021 and its general objective Propose a program with the development of entrepreneurial skills that will contribute to the personal growth of high school students in the 09D08 district of Guayaquil. The study was carried out with a population of 35 students and a non-probabilistic sample, the type of propositive-descriptive research, qualitative approach and nonexperimental design, the survey technique was applied, and as an instrument a questionnaire consisting of twenty items, applying in each one of them, five alternatives with the Lickert assessment scale. The collected data were processed in the SPSS software, a content matrix was made for the validation of the questionnaire, it was validated through the judgment of 5 experts, the reliability was .928 , the Cronbach's Alpha coefficient was used. The results of the investigation showed that in general, the personal growth of high school students from District 09D08 of an Educational Institution in Guayaquil, is valued as deficient. This was evidenced through the dimensions of the evaluated variable (Table 12), of $100 \%$ of the students surveyed, $68.6 \%$ show difficulty in resolving conflicts, $84.30 \%$ are not empathetic, $68.6 \%$ have low self-esteem, $74,3 \%$ do not have the ability to influence people, $68.5 \%$ show difficulty in making decisions, $68.60 \%$ show difficulty in the development of skills and competencies, $80.0 \%$ lack selfconfidence, even $74.20 \%$ have no desire to improve. The proposed program of personal growth through the development of entrepreneurial competencies as an alternative to improve and overcome limitations considerably and improve their well-being
\end{abstract}

Keywords: interpersonal relationships, empathy, decision making, self-realization, leadership.

\title{
1 INTRODUCCIÓN
}

En el trascurso del tiempo la humanidad pasa entre varios sucesos importantes, tanto económico, político sociales y tecnológicos, cada sujeto va requiriendo nuevos conocimientos y apoyos para su crecimiento, en la actualidad, una de las tendencias a nivel mundial, es formar jóvenes emprendedores, 
que tengan voluntad, percepción de oportunidades, habilidades para innovar, capaces de tener iniciativas propias, para el desarrollo de nuevas plazas de empleo, es un tema de gran utilidad, por la actual crisis laboral que vivimos.

Los adolescentes deben aprender a ajustarse a los cambios continuos ser diestros, capaces de innovar, autosuficientes, conllevándolos a una vida de bienestar personal, laboral, social u otros. Un grupo reducido de jóvenes desarrollan sus competencias y habilidades personales demostrando seguridad y creatividad, y, otros, en cambio presentan mucha dificultad, por lo que necesitan de apoyo constante.

En lo nacional dentro del contexto educativo uno de los desafíos más grande es el de formar adolescentes para que sean resilientes y sigan proyectándose al futuro con la posibilidad de lograr alcanzar las metas propuestas. Por tanto, la incorporación en el currículo educativo la asignatura de emprendimiento y gestión la finalidad es mejorar el estilo de vida del ser humano, de crear una cultura emprendedora para que los jóvenes se acepten así mismo como un ser visionario, con liderazgo, seguro de sí. Ministerio de educación del Ecuador (2015).

Dentro del sector educativo para el crecimiento o desarrollo personal de los adolescentes, el emprendimiento se ha convertido en una excelente herramienta para desarrollar creatividad, confianza en sí mismo, además las competencias y habilidades para arriesgarse, adaptarse y convertir las ideas en acciones. De acuerdo con Grebel, Pyka \& Hanusch (2003) el emprendimiento tiene un papel fundamental en la creación de nuevas fuentes de empleo, con la incorporación de nueva tecnología.

Si bien es cierto, el crecimiento o superación personal, es un proceso de transformación a través del desarrollo de las habilidades propias del individuo, mejorando las capacidades, toma de decisiones, aptitudes, actitudes, cualidades y acciones, permitiendo salir del estado de confort y alcance una mejor calidad de vida. "En el contorno personal, la competencia emprendedora ayuda a los jóvenes a hacer frente a los retos existentes, en el desarrollo de sus fortalezas y debilidades, y a preparar su autoconocimiento", afirma Planellas (2020).

La base de la problemática en el entorno local en la ciudad de Guayaquil, el aumento del desempleo juvenil a causa de la emergencia sanitaria, el coronavirus no solo desequilibro la economía del país, sino también las fuentes de trabajo, la educación y formación, los adolescente han sido tentados y captados por redes delincuenciales, sicariato, prostitución, tráfico y consumo de estupefacientes, alejándolos totalmente de sus sueños aspiraciones, anhelos, sin poder llegar a cumplir su proyecto de vida inicial, en esta situación adversa, muy pocos jóvenes desarrollaron habilidades y practicaron sus competencias emprendedoras, ante esta problemática, se ha creído conveniente aplicar la investigación que responde a las preguntas ¿Cuáles son las competencias emprendedoras que permitirán contribuir en el crecimiento personal de los estudiantes de bachillerato del distrito 09D08 de Guayaquil?. 
Velázquez (2018), asume, que el crecimiento o superación personal, es un proceso de transformación a través del desarrollo de las habilidades propias del individuo, mejorando las capacidades, toma de decisiones, aptitudes, actitudes, cualidades y acciones, permitiendo salir del estado de confort y alcance una mejor calidad de vida.

Los objetivos planteados en esta investigación son los siguientes: Objetivo general es Proponer un programa con el desarrollo de competencias emprendedoras que permitirán contribuir en el crecimiento personal de los estudiantes de bachillerato del distrito 09D08 de Guayaquil. Por consiguiente, como objetivos específicos en este estudio son los siguientes: Describir las características que tienen las relaciones interpersonales de los estudiantes de bachillerato del distrito 09D08 de Guayaquil; Conocer como son las Tomas de decisiones de los estudiantes de bachillerato del distrito 09D08 de Guayaquil; Identificar las características que presentan la autorrealización de los estudiantes de bachillerato del distrito 09D08 de Guayaquil. e Identificar las competencias emprendedoras que debe contener la propuesta de un programa de crecimiento personal para los estudiantes de bachillerato del distrito 09D08 de Guayaquil.

Durante las investigaciones realizadas, se encontró información relacionada con las variables de estudio, situándolo en el contexto internacional, En México, Silva (2020) en su artículo científico titulado "Estudio de las Capacidades y Perfil Emprendedor de Estudiantes de Nivel Medio Superior Dentro de un Contexto Rural" cuyo objetivo de investigación en conocer la influencia que ejerce la variable independiente Perfil emprendedor y variables de control (género, estado laboral y ocupación de la madre), sobre la variable dependiente Capacidad emprendedora...Los resultados muestran una relación significativa entre ambas variables, el estado laboral y la ocupación de la madre tiene un efecto negativo, mientras que el emprendimiento se ve afectado positivamente. Puedo concluir que este trabajo de investigación es de gran utilidad para mi trabajo de investigación dentro del principal hallazgo manifiesta que las mujeres no desarrollan la capacidad emprendedora a causa de la presión moral y social

En Perú Arauco \& Ramírez (2019) en su artículo titulado "Inteligencia emocional y autosuficiencia emprendedora y capacidad innovadora en universidades de la amazonia peruana", el objetivo central de la indagación "implantar la interacción existente entre la sabiduría emocional, la autoeficacia emprendedora y la capacidad innovadora en alumnos de ingeniería de las universidades de la amazonia peruana", estudio cuantitativo, de tipo transversal y correlacional, las herramientas usados en la autoeficacia emprendedora ha sido de Nobel, Jung y Ehrlich, y, para la medición de la capacidad innovadora de Armitage y Conner, además se usó el test de inteligencia emocional de Shutte...Como conclusión este estudio ha permitido demostrar que entre mayor inteligencia emocional aumentara la capacidad creativa, así mismo entre mayor autoeficacia mayor capacidad creativa en los estudiantes 
universitarios siendo evidente que las variables de este trabajo investigativo son indispensables para el desarrollo de las capacidades creativas, principalmente en tiempos de crisis.

Además, se puede enseñar nuestra interacción con la sociedad al instante de ser cordiales y de dejar independiente un asiento para una persona con propiedades especiales. Gómez (2013).

La teoría humanista se define como "un enfoque humanista asegura que los seres sintientes sean agentes libres con una mejor capacidad para usar simbología y pensar en términos abstractos". Philip (1997: 40), lo que podría decir que el individuo tiene la función de pensar y reorganizar su propio andamiaje y experiencias aprendidas para resolver problemas.

La teoría de la personalidad desarrollada por Carl Rogers citada por Hernández (2013), del crecimiento personal, enfocada a tomar en cuenta la soberanía humana, el auto juicio y la motivación, con la formulación de la teoría centrada en el consumidor definida como "Si la población recibe la independencia y el apoyo moral para desarrollar sus propias competencias, podrán ser capaces de resolver sus propias problemáticas, convirtiéndose en lo que quieren ser si ser criticados. Philip (1997: 42) se refiere a que una persona sana es una persona emocionalmente estable e independiente, porque cumplirá cada una de sus metas y objetivos.

La teoría fundamentada en oportunidades manifiesta que los emprendedores ejecutan cambios (como sostiene Schumpeter), sino también que saben aprovecharse de las oportunidades que se les presenta en el camino para crear Drucker (1985). Está claro en Drucker's Building Opportunity que los nuevos empresarios tienen en cuenta las posibilidades creadas para el cambio en lugar de los conflictos. Scholte, Teeffelen; y Verburg (2015). Scholte, Teeffelen; y Verburg (2015). Desde el punto de vista de Stevenson y Harmeling (1990) indican que, la gestión empresarial tiene como eje la búsqueda de oportunidades sin tener en cuenta los recursos. Para Schwartz \& Bilsky, (1987); Ros \& Grad, (1991); Grad, Ros, Alvero \& Torregrosa, (1993) \& Schwartz, (1992). Los valores personales son las pautas de cada persona para actuar ante cualquier circunstancia como guía de su vida definiendo su personalidad, estos valores individuales tienen flexibilidad y varían de acuerdo con el crecimiento personal, el poseer valores personales solidos permite el desarrollo de autodeterminación para enfrentar conflictos y superarlos.

\section{METODOLOGÍA}

El presente trabajo pertenece a un tipo de investigación proyectiva, intenta dar solución a la problemática encontrada, como alternativa ante la dificultad encontrada, se elaboró una propuesta de un programa de crecimiento personal a través del desarrollo de las competencias emprendedoras en los adolescentes de bachillerato. El proceso del trabajo investigativo se encuentra bajo el enfoque 
cuantitativo; se realizó un diagnóstico y la revisión bibliográfica que permitió la argumentación del problema y el diagnóstico del objeto de estudio.

Diseño de investigación no experimental, porque, parte del diagnóstico de la actual situación, basada en la observación de los fenómenos en el entorno natural para ser analizados, la investigación se construyó sobre la problemática encontrada para diseñar una propuesta de solución para lograr alcanzar los objetivos que se plantearon en la investigación. Hernández (2014),

La población según Arias (2006) "Es un conjunto finito o infinito de elementos con peculiaridades comunes para los cuales serán extensivas las conclusiones de la investigación”(p. 81). La población objeto de estudio estuvo conformada por treinta y cinco participantes entre las edades de 16 y 17 años.

Tabla $N^{\circ}$ 1. Distribución de la población

\begin{tabular}{lc}
\hline Paralelos & $\mathbf{N}^{\circ}$ de estudiantes \\
\hline A & 35 \\
& Fuente: Colegio RVR
\end{tabular}

Unidad de análisis. Está conformada por 20 estudiantes de bachillerato del distrito 09D08.

\section{RESULTADOS}

O.E.1. Describir las características que tienen las relaciones interpersonales de los estudiantes de bachillerato del distrito 09D08 de Guayaquil.

Tabla 3. Características de las Relaciones Interpersonales según su indicador la comunicación.

\begin{tabular}{|c|c|c|c|c|c|c|c|c|c|c|c|c|}
\hline & \multicolumn{2}{|c|}{ Siempre } & \multicolumn{2}{|c|}{$\begin{array}{c}\text { Casi } \\
\text { siempre }\end{array}$} & \multicolumn{2}{|c|}{$\begin{array}{c}\text { Muchas } \\
\text { veces }\end{array}$} & \multicolumn{2}{|c|}{$\begin{array}{c}\text { Casi } \\
\text { Nunca } \\
\end{array}$} & \multicolumn{2}{|c|}{ Nunca } & \multicolumn{2}{|c|}{ Total } \\
\hline & $\mathrm{N}$ & & $\mathrm{N}$ & & $\mathrm{N}$ & & $\mathrm{N}$ & & $\mathrm{N}$ & & $\mathrm{N}$ & \\
\hline & $\mathrm{O}$ & $\%$ & o & $\%$ & o & $\%$ & o & $\%$ & o & $\%$ & $\mathrm{O}$ & $\%$ \\
\hline $\begin{array}{l}\text { 1. ¿Puedes } \\
\text { comunicarte } \\
\text { con } \\
\text { facilidad con } \\
\text { las demás } \\
\text { personas? }\end{array}$ & 5 & $\begin{array}{c}14,3 \\
\%\end{array}$ & 5 & $\begin{array}{c}14,3 \\
\%\end{array}$ & 8 & $\begin{array}{c}22,9 \\
\%\end{array}$ & 8 & $\begin{array}{c}22,9 \\
\%\end{array}$ & 9 & $\begin{array}{l}25 \\
, 7 \\
\%\end{array}$ & $\begin{array}{l}3 \\
5\end{array}$ & $\begin{array}{c}100 \\
0 \%\end{array}$ \\
\hline $\begin{array}{l}\text { 2. ¿Afrontas } \\
\text { conversacion } \\
\text { es de forma } \\
\text { clara, sincera } \\
\text { y efectiva? }\end{array}$ & 2 & $\begin{array}{l}5,7 \\
\%\end{array}$ & 3 & $\begin{array}{c}8,6 \\
\%\end{array}$ & 7 & $\begin{array}{c}20,0 \\
\%\end{array}$ & $\begin{array}{l}1 \\
0\end{array}$ & $\begin{array}{c}28,6 \\
\%\end{array}$ & 13 & $\begin{array}{l}37 \\
, 1 \\
\%\end{array}$ & $\begin{array}{l}3 \\
5\end{array}$ & $\begin{array}{l}100, \\
0 \%\end{array}$ \\
\hline
\end{tabular}


Figura No 3

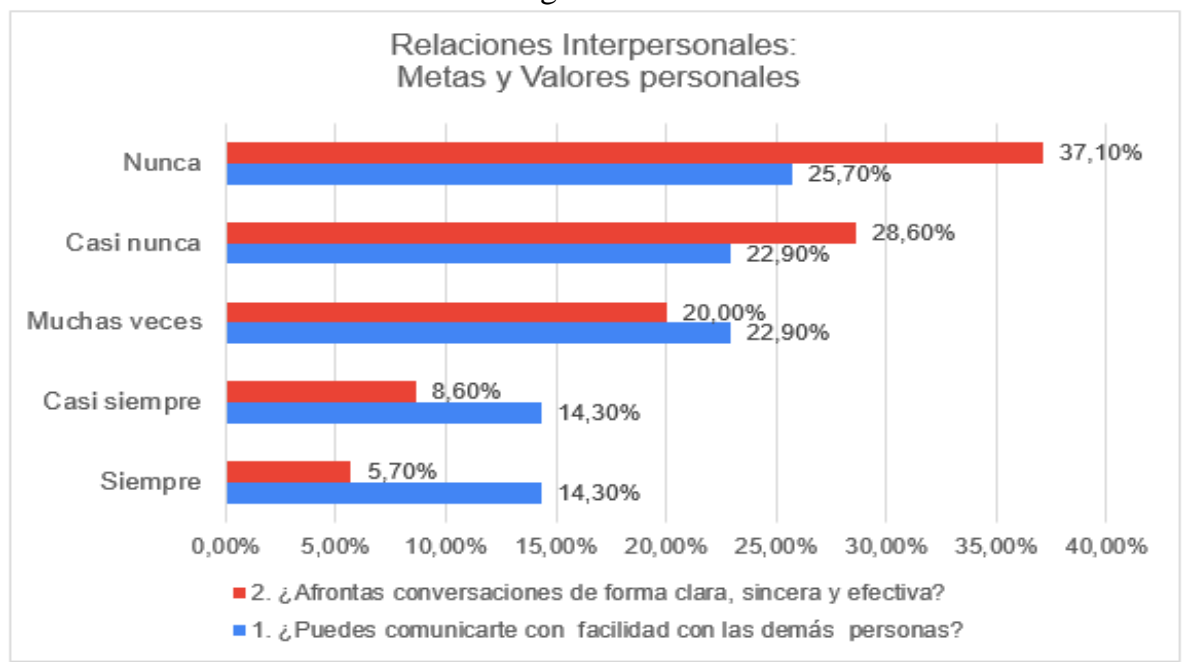

Fuente: Los porcentajes obtenidos son de la encuesta aplicada

Según la figura $\mathrm{N}^{\circ} 4$, los datos obtenidos de la variable relaciones personales, según su indicador la comunicación se ha logrado determinar, que, del 100\% de los estudiantes encuestados el 65,7\% se les dificulta enfrentar conversaciones de forma clara y sencilla; luego un $48.6 \%$ de los encuestados no pueden entablar conversación con otras personas que no sean de su entorno, a un $14.3 \%$ de los encuestados tiene facilidad para mantener conversaciones de forma clara y sencilla, y aun 28,6\% de los encuestados les resulta fácil entablar conversación con diferentes personas.

Tabla 4. Características de las Relaciones Interpersonales según su indicador la empatía.

\begin{tabular}{|c|c|c|c|c|c|c|c|c|c|c|c|c|}
\hline & \multicolumn{2}{|c|}{ Siempre } & \multicolumn{2}{|c|}{$\begin{array}{c}\text { Casi } \\
\text { siempre }\end{array}$} & \multicolumn{2}{|c|}{$\begin{array}{c}\text { Muchas } \\
\text { veces }\end{array}$} & \multicolumn{2}{|c|}{$\begin{array}{c}\text { Casi } \\
\text { Nunca }\end{array}$} & \multicolumn{2}{|c|}{ Nunca } & \multicolumn{2}{|c|}{ Total } \\
\hline & $\mathrm{N}$ & $\%$ & $\mathrm{~N}$ & $\%$ & No & $\%$ & $\mathrm{~N}$ & $\%$ & No & $\%$ & No & $\%$ \\
\hline & $\mathrm{O}$ & & o & & & & O & & & & & \\
\hline $\begin{array}{l}\text { 3.¿Cuándo } \\
\text { escuchas los } \\
\text { problemas de } \\
\text { otra persona, } \\
\text { tú mente } \\
\text { tiende a } \\
\text { intentar } \\
\text { buscar } \\
\text { soluciones? }\end{array}$ & 2 & $\begin{array}{c}5, \\
7 \\
\%\end{array}$ & 2 & $5,7 \%$ & 5 & $\begin{array}{c}14,3 \\
\%\end{array}$ & 12 & $\begin{array}{l}34, \\
3 \%\end{array}$ & 14 & $\begin{array}{l}40 \\
0 \%\end{array}$ & 35 & $\begin{array}{l}100 \\
0 \%\end{array}$ \\
\hline $\begin{array}{l}\text { 4. ¿Muestras } \\
\text { habilidades } \\
\text { para } \\
\text { interactuar } \\
\text { con las } \\
\text { personas, } \\
\text { adecuándote a } \\
\text { la manera de } \\
\text { ser del otro? }\end{array}$ & 2 & $\begin{array}{c}5, \\
7 \\
\%\end{array}$ & 2 & $5,7 \%$ & 7 & $\begin{array}{c}20,0 \\
\%\end{array}$ & 10 & $\begin{array}{l}28, \\
6 \%\end{array}$ & 14 & $\begin{array}{l}40 \\
0 \%\end{array}$ & 35 & $\begin{array}{l}100 \\
0 \%\end{array}$ \\
\hline
\end{tabular}

Fuente: Cuestionario aplicada a los estudiantes de bachillerato del distrito 09D08 de Guayaquil 
Figura No 5

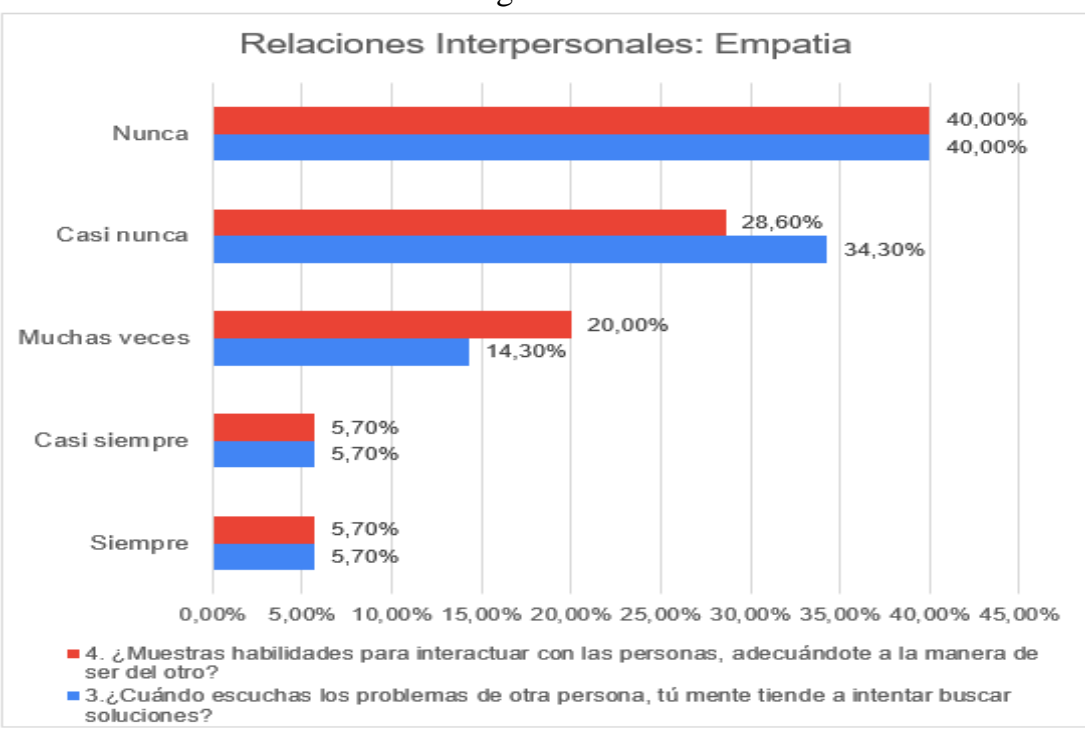

Fuente: Los porcentajes obtenidos son de la encuesta aplicada

Según la figura $\mathrm{N}^{\circ}$ 5. los datos obtenidos de la variable relaciones personales, según su indicador la empatía se ha logrado determinar, que, del $100 \%$ de los estudiantes encuestados el 68,60\% no son empático con los demás; luego un $84.3 \%$ de los encuestados no tiene habilidad para interactuar, adecuándose a la forma de ser de las otras personas, a un $11.40 \%$ de los encuestados son empático con las demás personas, y aun 11,4\% de los encuestados tienen habilidades para interactuar y adecuándose a la forma de pensar con los demás personas.

Tabla 5. Características de las Relaciones Interpersonales según su indicador asertivo.

\begin{tabular}{|c|c|c|c|c|c|c|c|c|c|c|c|c|}
\hline & \multicolumn{2}{|c|}{ Siempre } & \multicolumn{2}{|c|}{$\begin{array}{c}\text { Casi } \\
\text { siempre } \\
\end{array}$} & \multicolumn{2}{|c|}{$\begin{array}{c}\text { Muchas } \\
\text { veces }\end{array}$} & \multicolumn{2}{|c|}{ Casi nunca } & \multicolumn{2}{|c|}{ Nunca } & \multicolumn{2}{|c|}{ Total } \\
\hline & $\begin{array}{l}\mathrm{N} \\
\mathrm{o}\end{array}$ & $\%$ & $\begin{array}{l}\mathrm{N} \\
\mathrm{O}\end{array}$ & $\%$ & $\begin{array}{l}\mathrm{N} \\
\mathrm{O}\end{array}$ & $\%$ & $\begin{array}{l}\mathrm{N} \\
\mathrm{O}\end{array}$ & $\%$ & $\begin{array}{l}\mathrm{N} \\
\mathrm{O}\end{array}$ & $\%$ & $\begin{array}{l}\mathrm{N} \\
\mathrm{O}\end{array}$ & $\%$ \\
\hline $\begin{array}{l}5 . \quad \text { ¿Cuándo } \\
\text { estas } \\
\text { desempeñándo } \\
\text { te en algo } \\
\text { difícil o } \\
\text { desafiante, me } \\
\text { siento confiado } \\
\text { en mí triunfo? }\end{array}$ & 5 & $\begin{array}{c}14,3 \\
\%\end{array}$ & 3 & $\begin{array}{c}8,6 \\
\%\end{array}$ & 3 & $\begin{array}{l}8,6 \\
\%\end{array}$ & $\begin{array}{l}1 \\
0\end{array}$ & $\begin{array}{c}28,6 \\
\%\end{array}$ & 14 & $\begin{array}{l}40, \\
0 \%\end{array}$ & 35 & $\begin{array}{l}100, \\
0 \%\end{array}$ \\
\hline $\begin{array}{lr}6 . \quad \text { ¿Cuándo } \\
\text { estas } & \text { con } \\
\text { personas } & \\
\text { extrañas, } & \\
\text { puedes } & \\
\text { expresar } & \text { tus } \\
\text { opiniones } & \text { con } \\
\text { facilidad? } & \\
\end{array}$ & 5 & $\begin{array}{c}14,3 \\
\%\end{array}$ & 5 & $\begin{array}{c}14,3 \\
\%\end{array}$ & 4 & $\begin{array}{c}11,4 \\
\%\end{array}$ & $\begin{array}{l}1 \\
1\end{array}$ & $\begin{array}{c}31,4 \\
\%\end{array}$ & 10 & $\begin{array}{l}28, \\
6 \%\end{array}$ & 35 & $\begin{array}{c}100, \\
0 \%\end{array}$ \\
\hline
\end{tabular}


Figura No 6

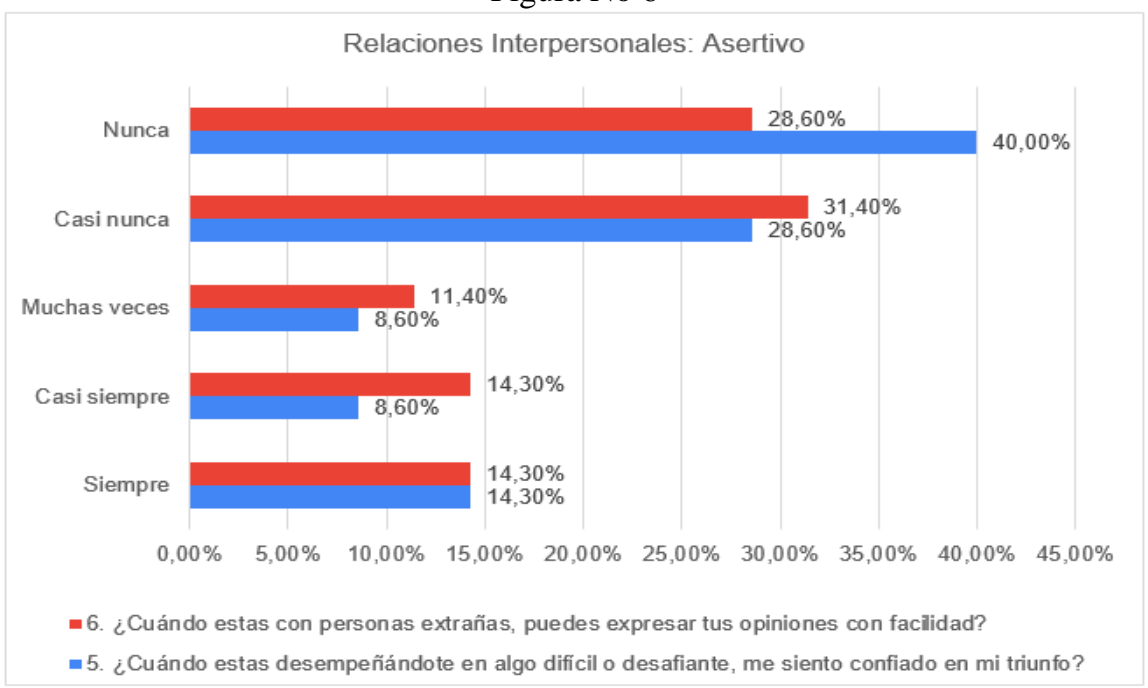

Fuente: Los porcentajes obtenidos son de la encuesta aplicada

Según la figura $\mathrm{N}^{\circ} 6$, los datos obtenidos de la variable relaciones personales, según su indicador asertivo se ha logrado determinar, que, del 100\% de los estudiantes encuestados el $60 \%$ de estos muestran dificultad para desempeñarse en algo difícil; luego un 68.6\% de los encuestados no pueden expresar sus opiniones con facilidad con otras personas que no sean de su entorno, mientras que el 28,60\% de los encuestados pueden lograr desempeñarse sin ningún tipo de dificultad, y un 22,90\% de los encuestados pueden expresar sus opiniones con facilidad con las demás personas.

Tabla 6. Características de las Relaciones Interpersonales según su indicador liderazgo.

\begin{tabular}{|c|c|c|c|c|c|c|c|c|c|c|c|c|}
\hline & \multicolumn{2}{|c|}{ Siempre } & \multicolumn{2}{|c|}{ Casi siempre } & \multicolumn{2}{|c|}{$\begin{array}{c}\text { Muchas } \\
\text { veces }\end{array}$} & \multicolumn{2}{|c|}{ Casi nunca } & \multicolumn{2}{|c|}{ Nunca } & \multicolumn{2}{|c|}{ Total } \\
\hline & No & $\%$ & No & $\%$ & No & $\%$ & No & $\%$ & No & $\%$ & No & $\%$ \\
\hline $\begin{array}{l}\text { 7. ¿Logras con } \\
\text { facilidad r que } \\
\text { otras personas } \\
\text { apoyen rus } \\
\text { recomendaciones } \\
\text { ? }\end{array}$ & 2 & $5,7 \%$ & 4 & $\begin{array}{c}11,4 \\
\%\end{array}$ & 7 & $\begin{array}{c}20,0 \\
\%\end{array}$ & 11 & $\begin{array}{c}31,4 \\
\%\end{array}$ & 11 & $\begin{array}{c}31,4 \\
\%\end{array}$ & 35 & $\begin{array}{l}100, \\
0 \%\end{array}$ \\
\hline $\begin{array}{l}8 . \\
\text { concentrarte en lo } \\
\text { primordial que } \\
\text { debes hacer un } \\
\text { día y encargar o } \\
\text { delegar tareas } \\
\text { necesarias } \\
\text { adicionales con } \\
\text { eficacia? }\end{array}$ & 2 & $5,7 \%$ & 4 & $\begin{array}{c}11,4 \\
\%\end{array}$ & 3 & $8,6 \%$ & 10 & $\begin{array}{c}28,6 \\
\%\end{array}$ & 16 & $\begin{array}{c}45,7 \\
\%\end{array}$ & 35 & $\begin{array}{l}100, \\
0 \%\end{array}$ \\
\hline
\end{tabular}

Fuente: Cuestionario aplicada a los estudiantes de bachillerato del distrito 09D08 de Guayaquil 
Figura $\mathrm{N}^{\circ} 7$

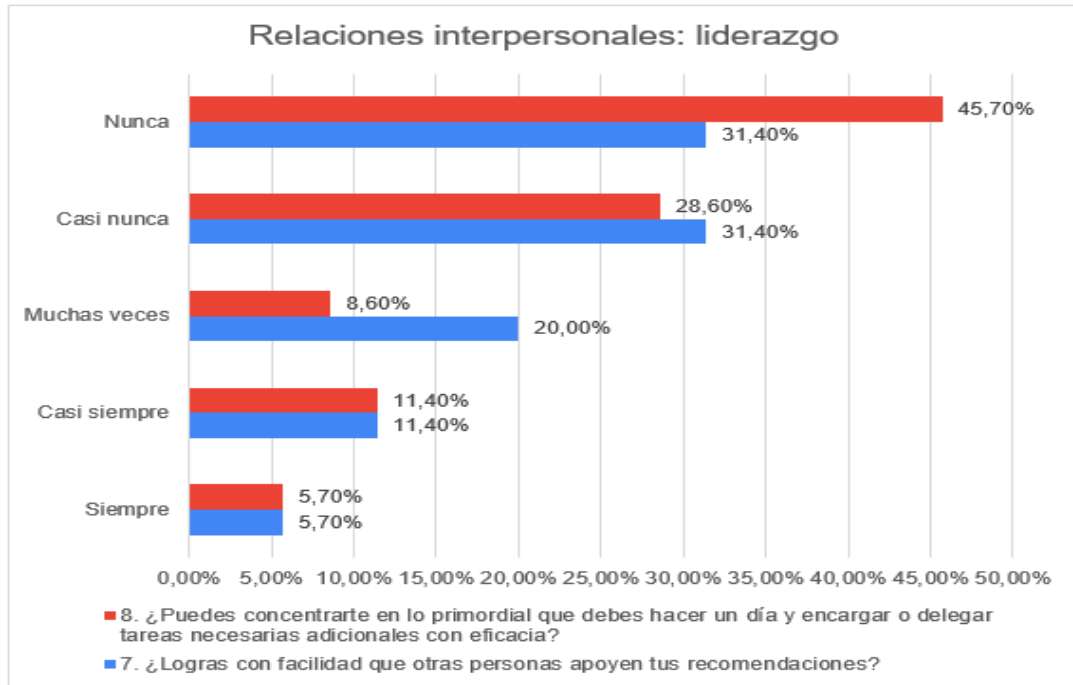

Fuente: Los porcentajes obtenidos son de la encuesta aplicada

Según la figura $\mathrm{N}^{\circ} 7$, los datos obtenidos de la variable relaciones personales, según su indicador liderazgo se ha logrado determinar, que, del $100 \%$ de los estudiantes encuestados el 74,30\% no puede delegar tareas concentrase en lo que hace; luego un 62,80\% de los encuestados estos muestran dificultad para lograr que otras personas apoyen sus recomendaciones, mientras que el 17,10\% de los encuestados pueden delegar tareas, y un 17,10\% de los encuestados logran con facilidad que otras personas lo apoyen.

O.E.2. Conocer como son las Tomas de decisiones de los estudiantes de bachillerato del distrito 09D08 de Guayaquil, 2021.

Tabla 7. La Toma de decisiones según sus indicadores objetivos personales y habilidades del pensamiento.

\begin{tabular}{|c|c|c|c|c|c|c|c|c|c|c|c|c|}
\hline & \multicolumn{2}{|c|}{ Siempre } & \multicolumn{2}{|c|}{$\begin{array}{c}\text { Casi } \\
\text { siempre }\end{array}$} & \multicolumn{2}{|c|}{$\begin{array}{c}\text { Muchas } \\
\text { veces }\end{array}$} & \multicolumn{2}{|c|}{ Casi nunca } & \multicolumn{2}{|c|}{ Nunca } & \multicolumn{2}{|c|}{ Total } \\
\hline & $\mathrm{N}$ & & $\mathrm{N}$ & & $\mathrm{N}$ & & $\mathrm{N}$ & & $\mathrm{N}$ & & $\mathrm{N}$ & \\
\hline & $\mathrm{o}$ & $\%$ & o & $\%$ & o & $\%$ & o & $\%$ & o & $\%$ & o & $\%$ \\
\hline $\begin{array}{l}\text { 9. ¿Cuándo te } \\
\text { planteas lo que } \\
\text { quieres lograr, no } \\
\text { te detienes hasta } \\
\text { alcanzarlo? }\end{array}$ & 3 & $\begin{array}{c}8,6 \\
\%\end{array}$ & 5 & $\begin{array}{c}14,3 \\
\%\end{array}$ & 6 & $\begin{array}{c}17,1 \\
\%\end{array}$ & 8 & $\begin{array}{c}22,9 \\
\%\end{array}$ & $\begin{array}{l}1 \\
3\end{array}$ & $\begin{array}{c}37,1 \\
\%\end{array}$ & $\begin{array}{l}3 \\
5\end{array}$ & $100,0 \%$ \\
\hline $\begin{array}{l}10 . \text { ¿Cuándo te } \\
\text { trazas un camino, } \\
\text { lo sigues aunque } \\
\text { demores en ver los } \\
\text { resultados? }\end{array}$ & 3 & $\begin{array}{l}8,6 \\
\%\end{array}$ & 3 & $8,6 \%$ & 5 & $\begin{array}{c}14,3 \\
\%\end{array}$ & $\begin{array}{l}1 \\
3\end{array}$ & $\begin{array}{c}37,1 \\
\%\end{array}$ & $\begin{array}{l}1 \\
1\end{array}$ & $\begin{array}{c}31,4 \\
\%\end{array}$ & $\begin{array}{l}3 \\
5\end{array}$ & $100,0 \%$ \\
\hline $\begin{array}{l}\text { 11. ¿Se las ingenia } \\
\text { en encontrar la } \\
\text { forma de obtener } \\
\text { lo que quieres sin } \\
\text { perjudicar a otros? }\end{array}$ & 2 & $\begin{array}{c}5,7 \\
\%\end{array}$ & 4 & $\begin{array}{c}11,4 \\
\%\end{array}$ & 5 & $\begin{array}{c}14,3 \\
\%\end{array}$ & $\begin{array}{l}1 \\
4\end{array}$ & $\begin{array}{c}40,0 \\
\%\end{array}$ & $\begin{array}{l}1 \\
0\end{array}$ & $\begin{array}{c}28,6 \\
\%\end{array}$ & $\begin{array}{l}3 \\
5\end{array}$ & $100,0 \%$ \\
\hline 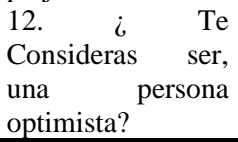 & 1 & $\begin{array}{c}2,9 \\
\%\end{array}$ & 2 & $5,7 \%$ & 4 & $\begin{array}{c}11,4 \\
\%\end{array}$ & $\begin{array}{l}1 \\
2\end{array}$ & $\begin{array}{c}34,3 \\
\%\end{array}$ & $\begin{array}{l}1 \\
6\end{array}$ & $\begin{array}{c}45,7 \\
\%\end{array}$ & $\begin{array}{l}3 \\
5\end{array}$ & $100,0 \%$ \\
\hline
\end{tabular}

Fuente: Cuestionario aplicada a los estudiantes de bachillerato del distrito 09D08 de Guayaquil 
Figura $\mathrm{N}^{\circ} 8$.

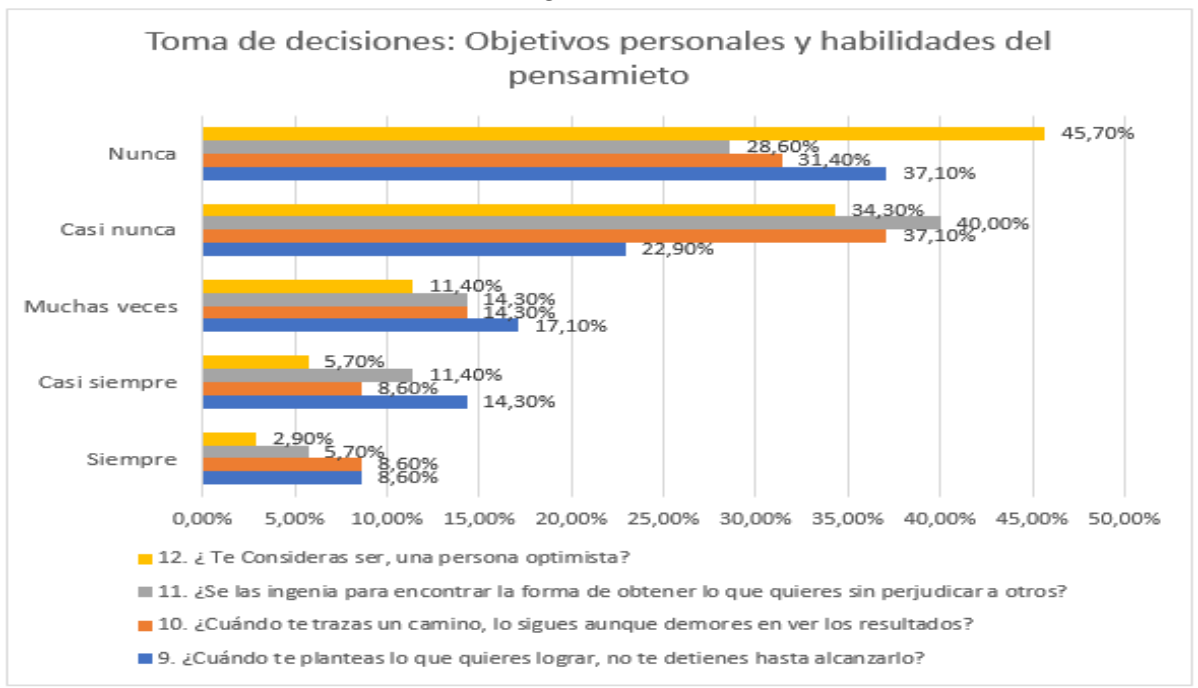

Fuente: Los porcentajes obtenidos son de la encuesta aplicada

Según la figura $\mathrm{N}^{\circ} 8$, los datos obtenidos de la variable relaciones personales, según su indicador objetivos personales y habilidades del pensamiento se ha logrado determinar, que, del 100\% de los estudiantes encuestados el $80 \%$ de estos muestran dificultad en lograr alcanzar lo que desean; luego un $68.6 \%$ de los encuestados abandonan su camino trazado y no lograr ver los resultados, mientras que el $68.5 \%$ de los encuestados no pueden ingeniársela para obtener lo que quieren, en consecuencia el $60 \%$ de los encuestados se consideran que no son personas optimistas, y un 8,60\% logran alcanzar lo deseado , así mismo el 17,10 de los encuestados logran continuar en el camino trazado por ellos, el 17.20\% de los encuestados se ingenian para obtener lo que quieren, y el $22.90 \%$ de los encuestados se consideran personas optimistas

0.E.3. Identificar las características que presentan la autorrealización de los estudiantes de bachillerato del distrito 09D08 de Guayaquil, 2021.

Tabla 8. Características de autorrealización según su indicador autonomía.

\begin{tabular}{|c|c|c|c|c|c|c|c|c|c|c|c|c|}
\hline & \multicolumn{2}{|c|}{ Siempre } & \multicolumn{2}{|c|}{ Casi siempre } & \multicolumn{2}{|c|}{ Muchas veces } & \multicolumn{2}{|c|}{ Casi nunca } & \multicolumn{2}{|c|}{ Nunca } & \multicolumn{2}{|c|}{ Total } \\
\hline & $\mathrm{N}$ & & $\mathrm{N}$ & & $\mathrm{N}$ & & $\mathrm{N}$ & & $\mathrm{N}$ & & $\mathrm{N}$ & \\
\hline & $\mathrm{o}$ & $\%$ & o & $\%$ & $\mathrm{o}$ & $\%$ & o & $\%$ & $\mathrm{o}$ & $\%$ & 0 & $\%$ \\
\hline $\begin{array}{l}\text { 13. ¿Puedes tomar } \\
\text { decisiones cuando } \\
\text { es necesario, sin } \\
\text { dilatar las cosas (no } \\
\text { deja para mañana lo } \\
\text { que tiene que } \\
\text { resolver)? }\end{array}$ & 6 & $\begin{array}{c}17,1 \\
\%\end{array}$ & 3 & $8,6 \%$ & 10 & $28,6 \%$ & 3 & $\begin{array}{c}8,6 \\
\%\end{array}$ & $\begin{array}{l}1 \\
3\end{array}$ & $37,1 \%$ & $\begin{array}{l}3 \\
5\end{array}$ & $100,0 \%$ \\
\hline $\begin{array}{lr}\text { 14. iTienes } & \text { la } \\
\text { capacidad } & \text { de } \\
\text { enfrentar } & \text { los } \\
\text { problemas } & \text { sin } \\
\text { acobardarte, ante } \\
\text { los fracasos } \\
\text { dificultades? }\end{array}$ & 2 & $5,7 \%$ & 4 & $11,4 \%$ & 6 & $17,1 \%$ & 9 & $\begin{array}{c}25,7 \\
\%\end{array}$ & $\begin{array}{l}1 \\
4\end{array}$ & $40,0 \%$ & $\begin{array}{l}3 \\
5\end{array}$ & $100,0 \%$ \\
\hline
\end{tabular}

Fuente: Cuestionario aplicada a los estudiantes de bachillerato del distrito 09D08 de Guayaquil 
Figura $\mathrm{N}^{\circ} 9$

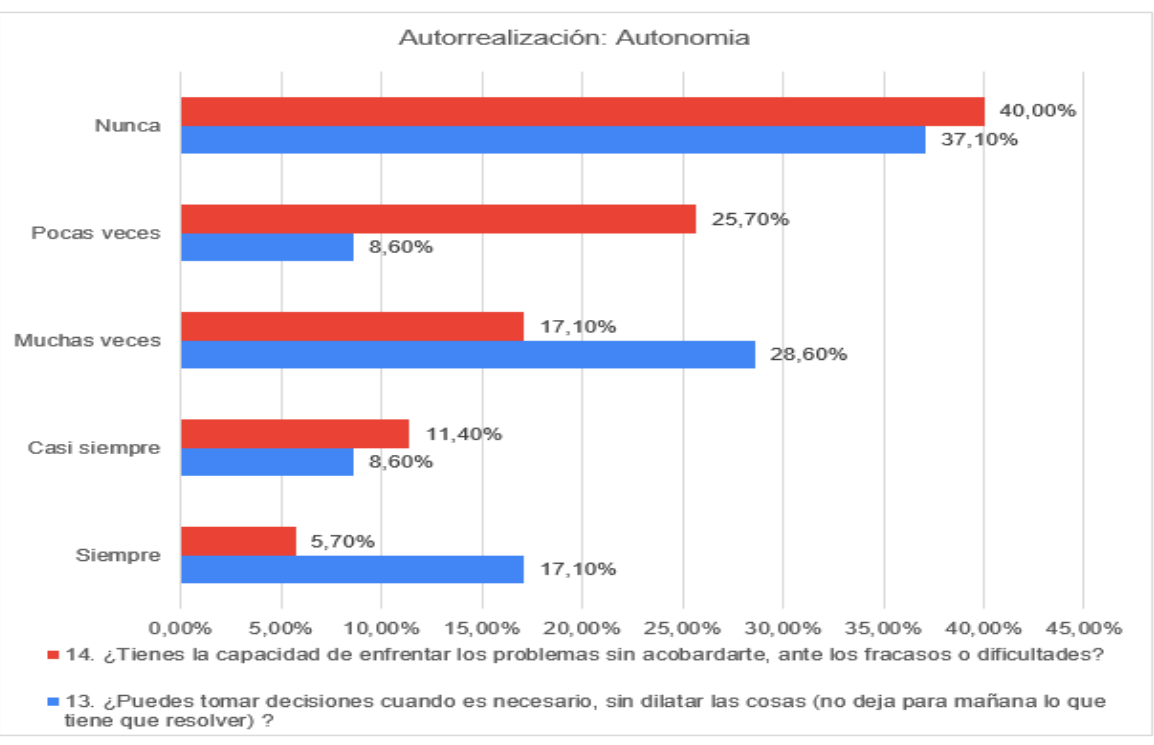

Fuente: Los porcentajes obtenidos son de la encuesta aplicada

Según la figura $\mathrm{N}^{\circ}$ 9, los datos obtenidos de la variable autorrealización, según su indicador Autonomía se ha logrado determinar, que, del $100 \%$ de los estudiantes encuestados el $65,7 \%$ de estos se les dificulta en el momento de tomar daciones; luego un $45.70 \%$ de los encuestados no pueden enfrentar problemas ante las dificultades, mientras que el $17.1 \%$ de los encuestados no tienen dificultas para la toma de decisiones, y un $25,7 \%$ de los encuestados pueden enfrentar problemas sin dificultad.

Tabla 9. Características de autorrealización según su indicador oportunidad.

\begin{tabular}{|c|c|c|c|c|c|c|c|c|c|c|c|c|}
\hline & \multicolumn{2}{|c|}{ Siempre } & \multicolumn{2}{|c|}{$\begin{array}{c}\text { Casi } \\
\text { siempre }\end{array}$} & \multicolumn{2}{|c|}{$\begin{array}{c}\text { Muchas } \\
\text { veces }\end{array}$} & \multicolumn{2}{|c|}{ Casi nunca } & \multicolumn{2}{|c|}{ Nunca } & \multicolumn{2}{|c|}{ Total } \\
\hline & $\begin{array}{l}\mathrm{N} \\
\mathrm{O}\end{array}$ & $\%$ & $\begin{array}{l}\mathrm{N} \\
\mathrm{o}\end{array}$ & $\%$ & $\begin{array}{l}\mathrm{N} \\
\mathrm{O}\end{array}$ & $\%$ & $\begin{array}{l}\mathrm{N} \\
\mathrm{O}\end{array}$ & $\%$ & $\begin{array}{l}\mathrm{N} \\
\mathrm{O}\end{array}$ & $\%$ & No & $\%$ \\
\hline $\begin{array}{l}\text { 15. ¿Disfrutas } \\
\text { y buscas las } \\
\text { oportunidades } \\
\text { de probar } \\
\text { cosas nuevas? }\end{array}$ & 4 & $\begin{array}{c}11,4 \\
\%\end{array}$ & 5 & $14,3 \%$ & 4 & $\begin{array}{c}11,4 \\
\%\end{array}$ & 8 & $22,9 \%$ & $\begin{array}{l}1 \\
4\end{array}$ & $\begin{array}{c}40,0 \\
\%\end{array}$ & 35 & $100,0 \%$ \\
\hline $\begin{array}{lr}16 . \quad ¿ C u a ́ n d o \\
\text { enfrentas un } \\
\text { problema } \\
\text { difícil, } \\
\text { inviertes gran } \\
\text { cantidad de } \\
\text { tiempo en } \\
\text { encontrar una } \\
\text { solución? }\end{array}$ & 2 & $\begin{array}{c}5,7 \\
\%\end{array}$ & 2 & $5,7 \%$ & 8 & $\begin{array}{c}22,9 \\
\%\end{array}$ & $\begin{array}{l}1 \\
1\end{array}$ & $31,4 \%$ & $\begin{array}{l}1 \\
2\end{array}$ & $\begin{array}{c}34,3 \\
\%\end{array}$ & 35 & $100,0 \%$ \\
\hline
\end{tabular}

Fuente: Cuestionario aplicada a los estudiantes de bachillerato del distrito 09D08 de Guayaquil 
Figura $N^{\circ} 10$

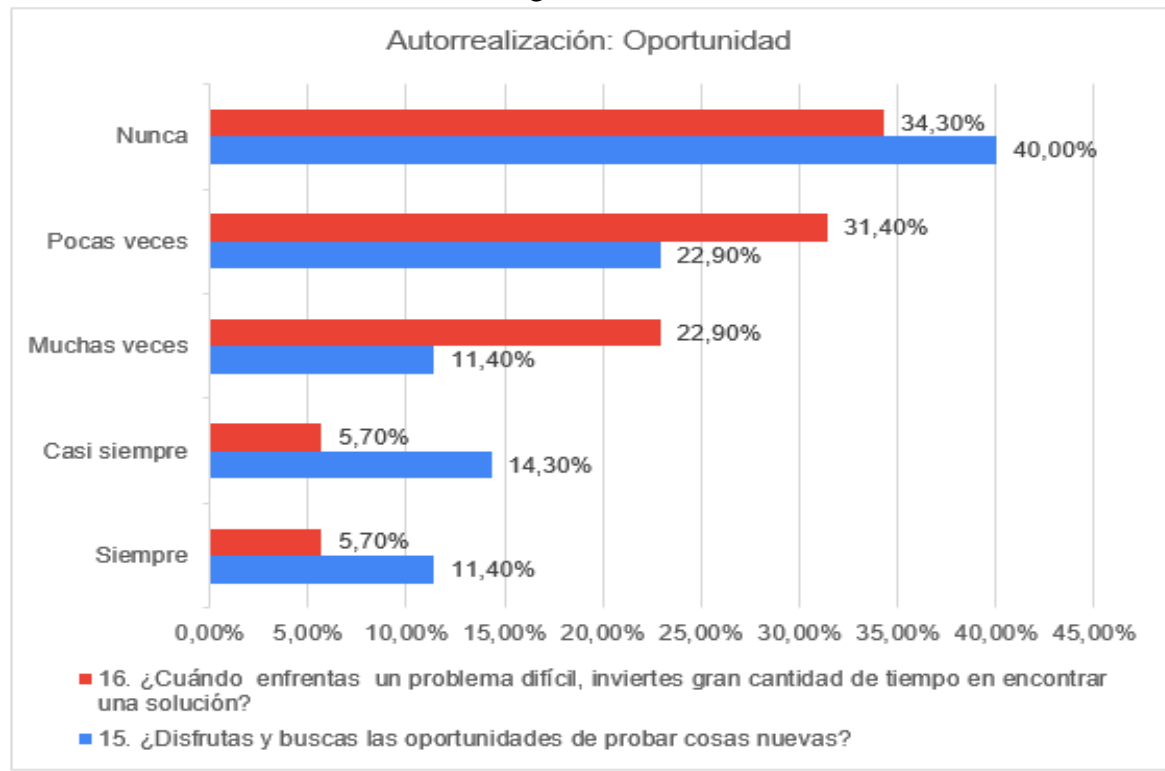

Fuente: Los porcentajes obtenidos son de la encuesta aplicada

Según la figura $\mathrm{N}^{\circ} 10$, los datos obtenidos de la variable autorrealización, según su indicador oportunidad se ha logrado determinar, que, del 100\% de los estudiantes encuestados el $65.7 \%$ de estos se les dificulta en la búsqueda de nuevas oportunidades, mientras que el 62.9\% de los encuestados no pueden invertir el tiempo necesario para encontrar solución frente a los problemas, a un 11,40 de los encuestados se les facilita la búsqueda de oportunidades, y un $25,7 \%$ de los encuestados invierten su tiempo en búsqueda de solución frente a los problemas que se le presenten.

Tabla 10. Características de autorrealización según su indicador deseos de superación

\begin{tabular}{|c|c|c|c|c|c|c|c|c|c|c|c|}
\hline \multicolumn{2}{|c|}{ Siempre } & \multicolumn{2}{|c|}{$\begin{array}{c}\text { Casi } \\
\text { siempre }\end{array}$} & \multicolumn{2}{|c|}{ Muchas veces } & \multicolumn{2}{|c|}{ Casi nunca } & \multicolumn{2}{|c|}{ Nunca } & \multicolumn{2}{|c|}{ Total } \\
\hline No & $\%$ & N & $\%$ & No & $\%$ & No & $\%$ & No & $\%$ & No & $\%$ \\
\hline
\end{tabular}

17. $\quad$ ¿Si

alguien te

hablara de la

misma manera

que te hablas a

ti mismo,

¿seguirías

teniendo

buena relación

con esa

persona?

18. ¿Tomas

posesión $\quad \mathrm{y}$

responsabilida

d por cosas

que te

comprometes

a realizar?

$\begin{array}{cccccccccccc}4 & 11,4 & 3 & 8,6 \% & 2 & 5,7 \% & 6 & 17,1 \% & 20 & 57,1 & 35 & 100,0 \%\end{array}$

$\begin{array}{cccccccccccc}6 & 17,1 & 6 & 17,1 \% & 8 & 22,9 \% & 6 & 17,1 \% & 9 & 25,7 & 35 & 100,0 \%\end{array}$

Fuente: Cuestionario aplicada a los estudiantes de bachillerato del distrito 09D08 de Guayaquil 
Figura No 11

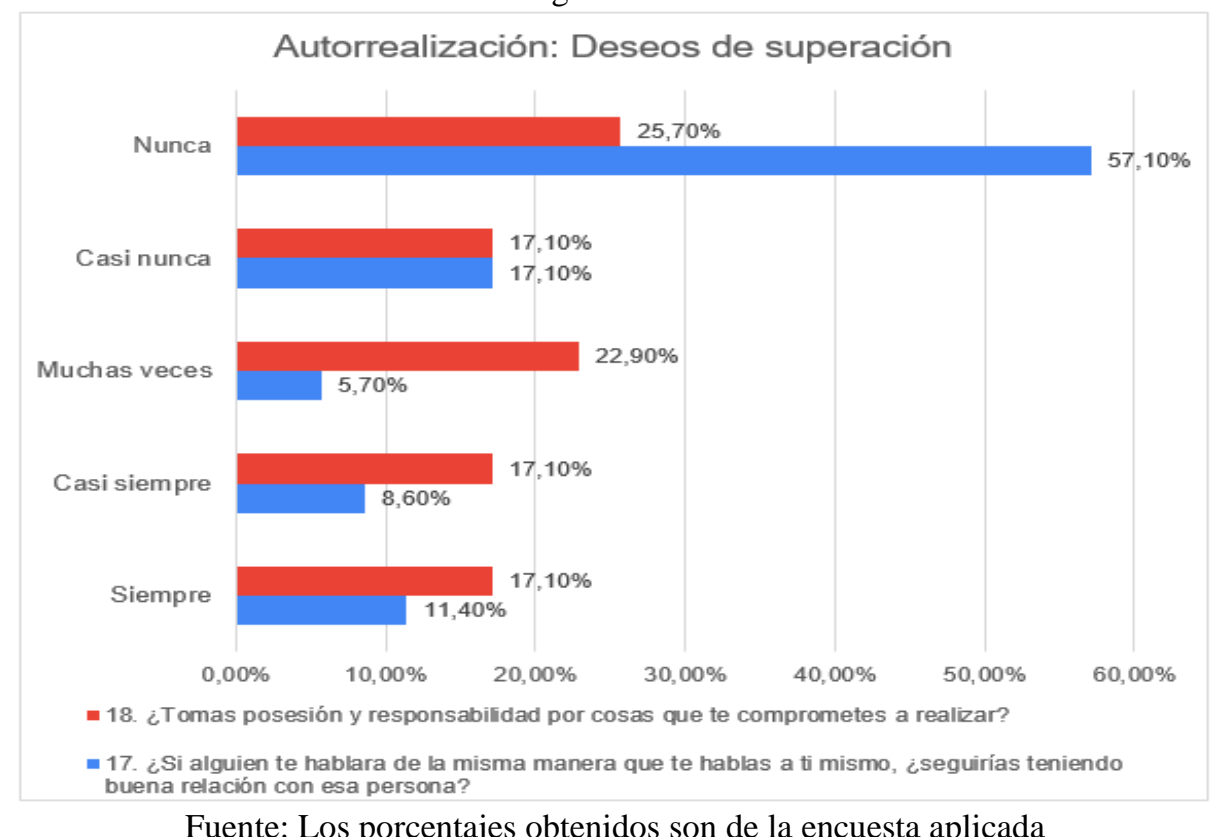

Fuente: Los porcentajes obtenidos son de la encuesta aplicada

Según la figura $\mathrm{N}^{\circ} 11$, los datos obtenidos de la variable autorrealización, según su indicador deseos de superación se ha logrado determinar, que, del 100\% de los estudiantes encuestados el $42.80 \%$ no son responsables y muestran dificultad a la hora de comprometerse con lo que se encuentran realizando, mientras que el $74,20 \%$ de los encuestados tienen dificultad en mantener buenas relaciones con otras personas, así mismo el $34.20 \%$ de los encuestados son responsables y no tienen dificultad a la hora de comprometerse, y un $20 \%$ de los encuestados mantienen buenas relaciones con otras personas.

Tabla 11. Características de autorrealización según su indicador metas y valores personales.

\begin{tabular}{|c|c|c|c|c|c|c|c|c|c|c|c|}
\hline \multicolumn{2}{|c|}{ Siempre } & \multicolumn{2}{|c|}{$\begin{array}{c}\text { Casi } \\
\text { siempre }\end{array}$} & \multicolumn{2}{|c|}{$\begin{array}{c}\text { Muchas } \\
\text { veces }\end{array}$} & \multicolumn{2}{|c|}{$\begin{array}{c}\text { Casi } \\
\text { Nunca }\end{array}$} & \multicolumn{2}{|c|}{ Nunca } & \multicolumn{2}{|c|}{ Total } \\
\hline $\begin{array}{l}\mathrm{N} \\
\mathrm{O}\end{array}$ & $\%$ & $\begin{array}{l}\mathrm{N} \\
\mathrm{O}\end{array}$ & $\%$ & $\begin{array}{c}\mathrm{N} \\
\mathrm{O}\end{array}$ & $\%$ & $\begin{array}{l}\mathrm{N} \\
\mathrm{O}\end{array}$ & $\%$ & $\begin{array}{l}\mathrm{N} \\
\mathrm{O}\end{array}$ & $\%$ & $\begin{array}{l}\mathrm{N} \\
\mathrm{O}\end{array}$ & $\%$ \\
\hline
\end{tabular}

19. ¿Tienes

valores

fundamentales

que influyen

$\begin{array}{cccccccccccc}8,6 & 4 & 11,4 \% & 6 & 17,1 \% & 7 & 20,0 & 1 & 42,9 & 3 & 100,0 \\ \% & 4 & 1,4 & 5 & \% & 5 & \%\end{array}$

decisiones?

20.¿Cuándo

no logras lo

deseado y

$\begin{array}{cccccccccccc}1 & 37,1 & 1 & 2,9 \% & 6 & 17,1 \% & 1 & 28,6 & 5 & 14,3 & 3 & 100,0 \\ 3 & \% & 1 & & & & & 0 & & & \end{array}$

fracasas,

piensas que

es el final?

Fuente: Cuestionario aplicada a los estudiantes de bachillerato del distrito 09D08 de Guayaquil 
Figura $\mathrm{N}^{\circ} 12$.

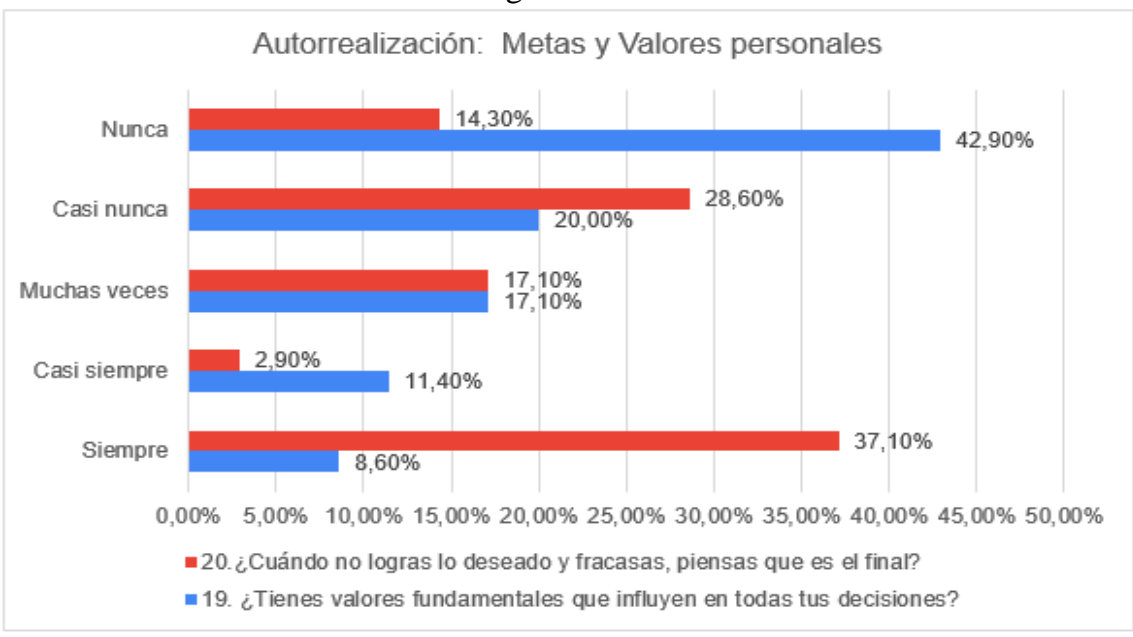

Fuente: Los porcentajes obtenidos son de la encuesta aplicada

Según la figura $\mathrm{N}^{\circ} 12$, los datos obtenidos de la variable autorrealización, según su indicador metas y valore, se em logrado determinar, que del 100\% de los estudiantes encuestados el 42,9\% piensan que, cuando no logran lo que desean es el final, mientras que el $62.90 \%$ de los encuestados no tienen valores fundamenta tales que influyen em sus decisiones, así mismo em $40 \%$ de los encuestados no piensan que, cuando no logran alcanzar lo deseado no es el final, y em $20 \%$ de los encuestados si tienen valore fundamentales que influyen em sus decisiones.

O.E.4. Identificar las mpreendedor mpreendedoras que debe contener la propuesta de em programa de crecimiento personal para los estudiantes de bachillerato del distrito 09D08 de Guayaquil,2021

Tabla 12. Factores que debe contener la propuesta em el programa de crecimiento personal para los estudiantes de bachillerato del distrito 09D08 de Guayaquil,2021

\begin{tabular}{|c|c|c|c|}
\hline Tabla & Dimensión & Factores & $\%$ \\
\hline \multirow{2}{*}{$\mathrm{N}^{\circ} 1$} & \multirow{2}{*}{$\begin{array}{l}\text { Relaciones Interpersonales: } \\
\text { Comunicación }\end{array}$} & Escasa capacidad para transmitir ideas & $48,60 \%$ \\
\hline & & Dificultad en el desarrollo de habilidades comunicativa. & $65,70 \%$ \\
\hline \multirow{2}{*}{$\mathrm{N}^{\circ} 2$} & \multirow[t]{2}{*}{ Relaciones Interpersonales: Empatía } & Dificultad para resolver conflictos & $68,60 \%$ \\
\hline & & Dificultad de relación de forma frecuente & $84,30 \%$ \\
\hline \multirow{2}{*}{$\mathrm{N}^{\circ} 3$} & \multirow[t]{2}{*}{ Relaciones Interpersonales: Asertivo } & Baja autoestima. & $68,60 \%$ \\
\hline & & Escasa fluidez al hablar. & $60,40 \%$ \\
\hline \multirow{2}{*}{$\mathrm{N}^{\circ} 4$} & \multirow[t]{2}{*}{ Relaciones Interpersonales: Liderazgo } & Escasa capacidad para delegar & $62.80 \%$ \\
\hline & & Escasa capacidad de influir en las personas & $74.30 \%$ \\
\hline \multirow{4}{*}{$\mathrm{N}^{\circ} 5$} & \multirow{4}{*}{$\begin{array}{l}\text { Toma de decisiones: Objetivos } \\
\text { personales y habilidades del pensamiento }\end{array}$} & Escasa habilidad de autoliderazgo & $60,00 \%$ \\
\hline & & Dificultad en la toma de decisiones & $68,50 \%$ \\
\hline & & Dificultad en el desarrollo de habilidades críticas & $68,60 \%$ \\
\hline & & Autoconfianza & $80,00 \%$ \\
\hline \multirow{2}{*}{$\mathrm{N}^{\circ} 6$} & \multirow{2}{*}{$\begin{array}{l}\text { Autorrealización: } \\
\text { Autonomía: }\end{array}$} & Carece de Decisiones personales & $45,70 \%$ \\
\hline & & Escasa capacidad para afrontar y tomar decisiones & $65,00 \%$ \\
\hline \multirow[b]{2}{*}{$\mathrm{N}^{\circ} 7$} & \multirow[b]{2}{*}{ Autorrealización: Oportunidad } & Dificultad para salir de la zona de confort & $62,90 \%$ \\
\hline & & $\begin{array}{l}\text { Dificultad de aprovechar las oportunidades que se les } \\
\text { presenta }\end{array}$ & $65.70 \%$ \\
\hline \multirow{2}{*}{$\mathrm{N}^{\circ} 8$} & \multirow{2}{*}{$\begin{array}{c}\text { Autorrealización: } \\
\text { Deseos de superación }\end{array}$} & Dificultad en el cambio de mentalidad y actitud. & $74.20 \%$ \\
\hline & & Dificultada para tazar nuevos logros & $42,10 \%$ \\
\hline \multirow{2}{*}{$\mathrm{N}^{\circ} 9$} & \multirow{2}{*}{$\begin{array}{l}\text { Autorrealización: } \\
\text { Metas y Valores }\end{array}$} & Carencia de valores & $62,90 \%$ \\
\hline & & Falta de estímulos, conductas y hábitos & $42,90 \%$ \\
\hline
\end{tabular}

Fuente: Elaboración Propia. 
De acuerdo a los resultados de las dimensiones de la variable evaluada (Tabla 12), del 100\% de los estudiantes encuestados, el 65,70\% no tienen buena comunicación, 68.6\% muestran dificultad para resolver conflictos, $84,30 \%$ no son empáticos, $68.6 \%$ tienen una baja autoestima, 74,3\% no tienen capacidad de influir en las personas, 68.5\% muestran dificultad para la toma de decisiones, 68.60\% muestran dificultad en el desarrollo de habilidades y competencias, $80.0 \%$ carece de autoconfianza, aun $74.20 \%$ no tienen deseos de superación. El programa propuesto de crecimiento personal a través del desarrollo de competencias emprendedoras como una alternativa para mejorar y superar las limitaciones de manera considerable y mejorar su bienestar.

\section{DISCUSIÓN}

García (2017) Su objetivo es fomentar la "conexión interpersonal" como un enriquecimiento recíproco de la base humana que conduce al acercamiento y conquista de la propia identidad personal en el camino del crecimiento y la auto creación, así como el desarrollo de la propia personalidad de la persona. El objetivo 1 Describir las características que tienen las relaciones interpersonales de los estudiantes de bachillerato. Respecto a los resultados de la encuesta de la Tabla 3 donde se destacan las principales características de las Relaciones Interpersonales según su indicador la comunicación, se logró determinar, que, del $100 \%$ de los estudiantes encuestados el 65,7\% consideran que tienen una mala habilidad comunicativa, el 20\% regularmente tienen habilidad para comunicarse, mientras que el 14,3\% tienen una habilidad comunicativita; luego un $48.6 \%$ no tienen capacidad para transmitir ideas, y aun $22,9 \%$ con regularidad pueden transmitir ideas, mientras que un $28,6 \%$ son capaces de transmitir ideas con facilidad. Suarez M. (2018) en su artículo "La comunicación como estrategia generadora de confianza en los emprendimientos".

Respecto a los resultados del Objetivo 2. Conocer como son las Tomas de decisiones de los estudiantes de bachillerato de la Tabla 6 que se realizó con el fin de identificar la Toma de decisiones según sus indicadores objetivos personales y habilidades del pensamiento, se ha logrado determinar, que, del $100 \%$ de los estudiantes encuestados el $80 \%$ de estos muestran dificultad en lograr alcanzar lo que desean; luego un 68.6\% de los encuestados abandonan su camino trazado y no lograr ver los resultados, mientras que el $68.5 \%$ de los encuestados no pueden ingeniársela para obtener lo que quieren, en consecuencia el $60 \%$ de los encuestados se consideran que no son personas optimistas, y un 8,60\% logran alcanzar lo deseado , así mismo el 17,10 de los encuestados logran continuar en el camino trazado por ellos, el $17.20 \%$ de los encuestados se ingenian para obtener lo que quieren, y el $22.90 \%$ de los encuestados se consideran personas optimistas. Este estudio concuerda con Hernández, Poveda \& Artola (2019) en su artículo titulado "Competencias personales de emprendedores y factores en la creación de empresas ecuatorianas" en este trabajo se logró evaluar y determinar las diez competencias personales 
entre ellas el trabajo en equipo, creatividad, toma de decisiones, liderazgo, tolerancia, etc. demostrando un bajo desarrollo en ambos grupos de estudio, con un índice de competencia $0,8 \%$ en los emprendedores activos y $0,62 \%$ en los emprendedores inactivos. Se observó que los jóvenes ecuatorianos tienen escasas capacitación de competencias personales. Las personas con baja capacidad de toma de decisiones no responden adecuadamente a una situación desafiante y, como resultado, no aceptan las consecuencias de sus decisiones. En el sustento teórico Sternberg (1997). Menciona que las personas emocionales exitosas pueden reflexionar sobre cómo y cuándo usar habilidades como la automotivación, el control de los impulsos, la independencia, la iniciativa y la perseverancia de manera efectiva, aprovechar al máximo sus habilidades, tener confianza en sí mismo y creer en su capacidad para lograr metas, superar las dificultades personales y equilibrar el pensamiento analítico y emocional.

Gómez Zatizábal (2011). Las competencias se entienden actualmente como acciones integradas que permiten identificar, interpretar, razonar y resolver problemas contextuales de forma adecuada y ética, saber qué hacer, saber hacerlo, saber. De acuerdo con los resultados del Objetivo 3. Identificar las características que presentan la autorrealización de los estudiantes, según la Tabla 7 que nos habla sobre las Características de autorrealización según su indicador autonomía, y de acuerdo a los datos obtenidos de la variable autorrealización, según su indicador Autonomía se ha logrado determinar, que, del 100\% de los estudiantes encuestados el 65,7\% de estos se les dificulta en el momento de tomar daciones; luego un $45.70 \%$ de los encuestados no pueden enfrentar problemas ante las dificultades, mientras que el $17.1 \%$ de los encuestados no tienen dificultas para la toma de decisiones, y un 25,7\% de los encuestados pueden enfrentar problemas sin dificultad, se determinó que no organizan su vida presente ni orientan sus esfuerzos en una dirección específica, por lo que existe la ausencia de la búsqueda de nuevas experiencias que posibiliten su realización personal. Este estudio concuerda con Vallejo. F (2017), en su estudio investigativo "Propuesta de un Modelo Formativo Integral de Emprendimiento Prospectivo a través de la Validación de los aprendizajes de la Carrera De Emprendimiento de la Universidad Católica de Santiago de Guayaquil", los resultados obtenidos de acuerdo a las variables de estudio: necesidad al éxito, autonomía e independencia, demostraron que el 28,2\% de la mujeres y el 24,6\% de hombres tienen necesidad al éxito, en cambio en la autonomía e independencia, se logró observar que son independientes en un $9,60 \%$ de los hombres y las mujeres un 9,5\% es notable que no existe diferencia entre ellos, el porcentaje de $56,3 \%$ los estudiantes que carecen de autonomía e independencia. En base teoría la autorrealización podría ser entonces una necesidad psicológica que surge después de que se han satisfecho las necesidades físicas y espirituales simples. Así es como alcanzamos nuestro potencial. Myers (2006).

De acuerdo con la teoría basada en oportunidades explica que los emprendedores realizan cambios (como sostiene Schumpeter) sino que aprovechan las oportunidades que se les presenta para crear Drucker (1985). Objetivo 3. Identificar las características que presentan la autorrealización de los estudiantes, 
según la Tabla 8; tomamos en cuenta características de autorrealización según su indicador oportunidad. según su indicador oportunidad se ha logrado determinar, que, del 100\% de los estudiantes encuestados el $65.7 \%$ de estos se les dificulta en la búsqueda de nuevas oportunidades, mientras que el 62.9\% de los encuestados no pueden invertir el tiempo necesario para encontrar solución frente a los problemas, a un 11,40 de los encuestados se les facilita la búsqueda de oportunidades, y un 25,7\% de los encuestados invierten su tiempo en búsqueda de solución frente a los problemas que se le presenten, cabe señalar, que los resultados obtenidos demuestran que la mayoría de los estudiantes no saben aprovechar las oportunidades, por no saber asumir los riesgo las dejan pasar de largo. En concordancia con Zumba, Mora \& Villacrés (2019), en su artículo "El emprendimiento como instrumento de desarrollo y su repercusión en el crecimiento personal y social de los jóvenes estudiantes universitario" se buscó impulsar a los jóvenes a emprender proyectos innovadores de impacto social a temprana edad, con el fin para desarrollar sus competencias, capacidades y habilidades, en saber aprovechar cuándo se presenta una oportunidad, determinar si es el momento más ventajoso para aprovecharla, determinar el beneficio que esa oportunidad puede traer a sus vidas y complete todas estas tareas antes de que se pierda. Como sustento teórico Vélez (2006). Las acciones se definen como aquellas actividades que comienzan con un proceso mental y terminan con la realización del acto correspondiente; una acción comienza en el momento en que un hombre propone mentalmente un objetivo y termina con la ejecución del acto correspondiente, con el objetivo, el plan mental, las opciones y el acto interviniendo todos en esta unidad de tiempo; una acción comienza en el momento en que un hombre propone mentalmente un objetivo y termina con la realización del acto correspondiente.

Según Mielina (2007). Con el fin de medir y evaluar la calidad de vida percibida, se debe identificar e implementar una solución a la dificultad de conceptualizar y medir este constructo. Hacer y verificar un instrumento para operacionalizar los diferentes aspectos involucrados en esta noción es un esfuerzo difícil que puede facilitarse mediante el uso de métodos estadísticos poderosos como el análisis factorial y los modelos de ecuaciones estructurales, ambos disponibles para los investigadores. Por esto Según los factores que debe contener la propuesta en el programa de crecimiento personal para los estudiantes de bachillerato del distrito 09D08 de Guayaquil,2021 presentada en la Tabla 11 se identificó que la mayoría de los estudiantes de secundaria interrogados en el área 09D08 de Guayaquil informan que no tienen objetivos, a pesar de que las metas son un componente importante del proceso de vida en sí.

\section{CONCLUSIONES}

Entre las características que tienen las relaciones interpersonales de los estudiantes, se aprecia que el 65,7\% de ellos tienen dificultad de comunicación (Tabla 3), además, el 68,60\% son carentes de empatía (Tabla 4); también, se refleja en la tabla 5 que, el 68,6 \% de los estudiantes evidencian carencia en la 
conducta asertiva. Por último, el 74,30 \% de los estudiantes no tienen liderazgo como se evidencia en la tabla 6.

El $68.6 \%$ de los estudiantes casi nunca pueden tomar decisiones, se les torna difícil como se evidencia en la tabla 7, además se conoció como son la toma decisiones de los estudiantes, en consecuencia, el $80 \%$ de los estudiantes no se encuentran preparados a perseguir y alcanzar sus objetivos (Tabla 7).

Las características que presentan la autorrealización de los estudiantes, se aprecia que, el 65,7\% de ellos carecen de autonomía (Tabla 8), así mismo el $65.7 \%$ de los estudiantes no aprovechan las oportunidades que se le presentan (Tabla 9), además, el 74,20\% de los estudiantes no tienen deseos de superación, tienen dificultad para logran alcanzar sus metas (Tabla 10). Por último, el 62.90\% de los estudiantes no tienen metas y valores personales sólidos como se evidencia en la tabla 11.

En el presente trabajo se identificó los factores o elementos que debe contener la propuesta, se pudo concluir que, las dimensiones de la variable evaluada (Tabla 12), el 65,70\% tiene dificultad comunicativa, $68.6 \%$ tiene problemas en resolver conflictos, $84,30 \%$ no son empáticos, $68.6 \%$ carecen de autoestima, 74,3\% no tienen poder de influir en otros, $68.5 \%$ tienen dificultad para la toma de decisiones, $68.60 \%$ carecen de habilidades y competencias, $80.0 \%$ carece de autoconfianza, y el $74.20 \%$ no tienen deseos de superación.

El programa propuesto debe incluir las competencias emprendedoras de relaciones interpersonales, toma de decisiones y la autorrealización, los cuales no se han desarrollado y su práctica permitirá contribuir en el crecimiento personal de los estudiantes. 


\section{REFERENCIA}

Anderson, W. (2001). Aprender a creer en usted mismo. Barcelona. Amat.

Annelissie, A. (2005). Emprendedorismo. Universidad NUR, Bolivia. Extraído el 12 de abril de 2018 desde en: http://produccionintelectual. Nur. Edu/archivos/emprendedurismo. Pdf.

Aponte, M. (2002). Factores condicionantes de la creación de empresas en Puerto Rico: un enfoque institucional. Universidad Autónoma de Barcelona.

Arboleda, M. (2011) El emprendimiento: Una respuesta al desempleo en Colombia. Recuperado de: https://www.usbcali.edu.co/sites/default/files/8_desempleo.pdf

Benet, William (1996). El libro de las virtudes. México: Vergara.

Chandler, G., \& Hanks, S. (1994). Founder Competence, the Environmnet, and Venture Performance. Entrepreneurship Theory \& Practice, 18(3), 77-89.

Dolabela, F. (2005). Taller del Emprendedor. Argentina: HomoSapiens. Universidad Nacional del Rosario.

Drucker, P. (1985). La Innovación y el empresario innovador. Buenos Aires:

Edhasa. EducarChile, portal de la educación (2008, Setiembre 1).

Educar para el emprendimiento. Extraído el 25 de febrero de 2018 desde http://www.educarchile.cl/Portal.Base/Web/VerContenido.aspx?GUID=5a31 d1cb-ac43-4258-9da278f0aedd5c41\&ID=187938.

Espinoza M (2018). Incidencia de los estilos de toma de decisiones en la relación entre liderazgo y desempeño organizacional en un grupo de directivos de Colombia, Ecuador y Perú. Liderazgo y Toma de Decisiones - Tesis PhD 2018.pdf (urosario.edu.co)

Guilford, J. (1967). The nature of human inteligence. New York: Mc Graw-Hill.

Hernández, R., Fernández, C., \& Baptista, P. (2010).

Hernández, S.; Fernández, C.; Baptista, L. (2016). Metodología de la Investigación.

Jarret, James L. (1991). The teaching of values. New York: Routledge.

Petra, T. Cortes, P. Herrera, M. Aburto, A. Farfan, A Cea

A self-assessment tool to evaluate the medical student's development and personal growth throughout his/her career. An International. En:

Abstract Book, Med Educ - AMEE (2013), pp. 593-594

Metodología de investigación. México: Mc GrawHill.

Méndez, R. (2009). El modelo de las tres necesidades del Dr. David McClelland.

Muñoz y Zabala (2018) Factores que influyen en la capacidad emprendedora del estudiantado. Articulo científico de la Universidad Central del Ecuador, publicado en la Revista Publicando, ISSN-e 1390-9304, 
Vol. 4, Nº 10, 1, 2017, págs. 406-419- Idioma: español.

Rogers (1902-1987). Teoría del crecimiento Personal.

Silva (2020) con su artículo científico titulado Estudio de las Capacidades y Perfil Emprendedor de Estudiantes de Nivel Medio Superior Dentro de un Contexto Rural. Articulo científico de la Universidad Autónoma de Aguascalientes, México. https://www.redalyc.org/journal/944/94465715004/html/

Marulanda Valencia, F. A., Montoya Restrepo, I. A., \& Vélez Restrepo, J. M. (2019). El Individuo y sus motivaciones en el proceso emprendedor. Universidad \& Empresa, 21(36), 149-174. 